\section{Trends in paediatric asthma hospitalisations - differences between neighbouring \\ countries}

Paediatric asthma hospitalisation trends in Finland and Sweden over the past 10 years are unclear. We investigated the incidence of paediatric asthma hospitalisations in both countries from 2005 to 2014, using Finland's National Hospital Discharge Register and Sweden's National Patient Register which cover all hospitalisations in the respective countries. During the study period, the incidence of paediatric asthma hospitalisations decreased by $51 \%$ in Finland, but remained stable in Sweden. In both countries, asthma hospitalisations decreased over time among children aged $0-4$ years, with unclear trends for older ages. The reasons for these intercountry differences are unclear, thus warranting future research.

\section{INTRODUCTION}

The global burden of asthma and allergic diseases is substantial. Yet, there appears to be a decrease in hospitalisations, mostly due to improved medication. ${ }^{1}{ }^{2}$ We previously reported an increasing incidence of paediatric anaphylaxis-related hospitalisations in Finland and Sweden, but which varied widely between the countries. ${ }^{3}$ With regard to paediatric asthma hospitalisations in these countries, the rates were comparable at the end of the last century. ${ }^{4}$ However, the trends of incidences over the past decade are unclear.

To this end, we aimed to investigate the incidence of paediatric asthma hospitalisations in Finland and Sweden from 2005 to 2014 .

\section{METHODS}

This study is based on data from two hospital patient registers: Finland's National Hospital Discharge Register and Sweden's National Patient Register. Both registers contain inpatient data including age, time of hospitalisation and diagnosis, from all hospitals in the respective countries.

All children aged 0-19 years old who were hospitalised with a primary diagnosis of asthma between 2005 and 2014 were identified by computer searches using the International Classification of Diseases, 10th Revision (ICD-10) codes. Our main outcome variable was the number of children hospitalised with a primary diagnosis of asthma and corresponding ICD-10 codes: J45 (asthma) and J46 (status asthmaticus). Consideration was given to sex and age groups (ages 0-4, 5-9, 10-14 and
15-19 years). As hospitalisations due to bronchiolitis may be a confounding factor, we also performed separate search for code J21. Given that wheeze due to viral infections may be miscoded as asthma in young children, we additionally examined 'small wheezers' by considering a combined code of J45-J46 and J21 (acute bronchiolitis) among children aged 0-4 years.

To compute the incidence of asthma hospitalisations, the annual age-specific midpopulations were obtained from Official Statistics of Finland and Statistics Sweden. The incidence of hospitalisations (per 10000 persons) was based on the results of the entire population of children in each country rather than cohort or sample-based estimates. The general incidence of asthma hospitalisations was analysed by Poisson regression analysis. Results were given as incidence rate ratios (IRR) with 95\% CIs. The statistical analyses were conducted with Stata V.13.0 (Stata).

\section{RESULTS}

From 2005 to 2014, paediatric asthma hospitalisations totalled 12565 in Finland and 28574 in Sweden. During this period, the incidence of asthma hospitalisations decreased in Finland, from 14.0 per 10000 person-years in 2005 to 6.8 in 2014 (IRR 0.936, 95\% CI 0.930 to 0.941). The total decrease in incidence was $51.4 \%$ in Finland. At the start of the study period, the incidence in Sweden (13.6 per 10000 person-years) approximated that seen in Finland and remained relatively stable by 2014 (IRR 0.994, 95\% CI 0.990 to 0.998 ) (figure $1 \mathrm{~A}$ ). In both countries, the majority of hospitalised children were boys (Finland: 65.6\%; Sweden 64.4\%). With consideration to gender, the same decreasing trend was seen in Finland, as was a stable trend in Sweden.

The majority of hospitalised children were aged 0-4 years (Finland: 70.0\%; Sweden: $81.6 \%$ ). Yet, asthma hospitalisations decreased over time among children aged $0-4$ years. The decrease in Finland was $60.3 \%$, from 40.3 per 10000 personyears (2005) to 16.0 per 10000 (2014) person-years. In Sweden, the decrease was $21.8 \%$, with corresponding incidences of 49.1 and 38.4 per 10000 person-years. Across other age groups, trends in the incidence of hospitalisations were less clear (figure 1B, C).

Between 2005 and 2014, hospitalisations with the primary diagnosis code $\mathrm{J} 21$ (acute bronchiolitis) totalled 37604 in Finland and 18932 in Sweden. The incidence of hospitalisations with this code was stable over time in both countries, although the incidence in Finland (27.3-38.0 per 10000 person-years) was notably higher than in Sweden (6.1-10.9). Children aged 0-4 years accounted nearly all of these hospitalisations (Finland: 97.1\%; Sweden: 99.1\%). In this age group, the incidence of hospitalisation for 'small wheezers' decreased in Finland from 168.3 per 10000 person-years in 2005 to 135.4 in 2014. In Sweden, the corresponding figures are 75.6 and 72.4 per 10000 person-years, respectively (figure 2).

In 2005, the incidence of hospitalisations for 'small wheezers' remained approximately steady in both countries through 2014 (figure 2).

\section{DISCUSSION}

From 2005 to 2014, the incidence of paediatric asthma hospitalisations decreased in Finland by $51 \%$. A similar decrease was not seen in Sweden. The overall decrease in hospitalisations in both countries was mainly due to fewer admissions of children aged $0-4$ years, who represented the majority of patients. Over the same period, the incidence of hospital admissions due to wheezing was higher in Finland than that in Sweden.

This study has some limitations. As data were based on ICD codes and hospitalisation statistics, consideration to treatments given in the emergency department (ED) or patient outcomes following discharge was not possible. Moreover, we cannot be certain if there were differences in diagnosing asthma between the countries.

Nonetheless, our findings are novel, as they describe the national trends over a 10 -year period of paediatric asthma hospitalisations in two well-defined populations. The major strength of this study is the use of national-level hospitalisation data from validated and well-established registers. ${ }^{5} 6$ Moreover, the use of standardised ICD-10 diagnostic coding permitted estimations of incidence and an international comparison.

Elsewhere, paediatric asthma hospitalisations have also decreased. ${ }^{2}$ Although earlier reports on paediatric asthma hospitalisations describe a similar decreasing incidence, they do not present trends over time or make comparisons between countries. ${ }^{8-10}$ Prior to 2000 , the incidence of paediatric asthma hospitalisations was comparable between Finland and Sweden. ${ }^{4}$ However, our findings indicate a decreasing incidence in Finland but not in Sweden. As the trend of incidences of hospital admissions due to wheezing is stable 

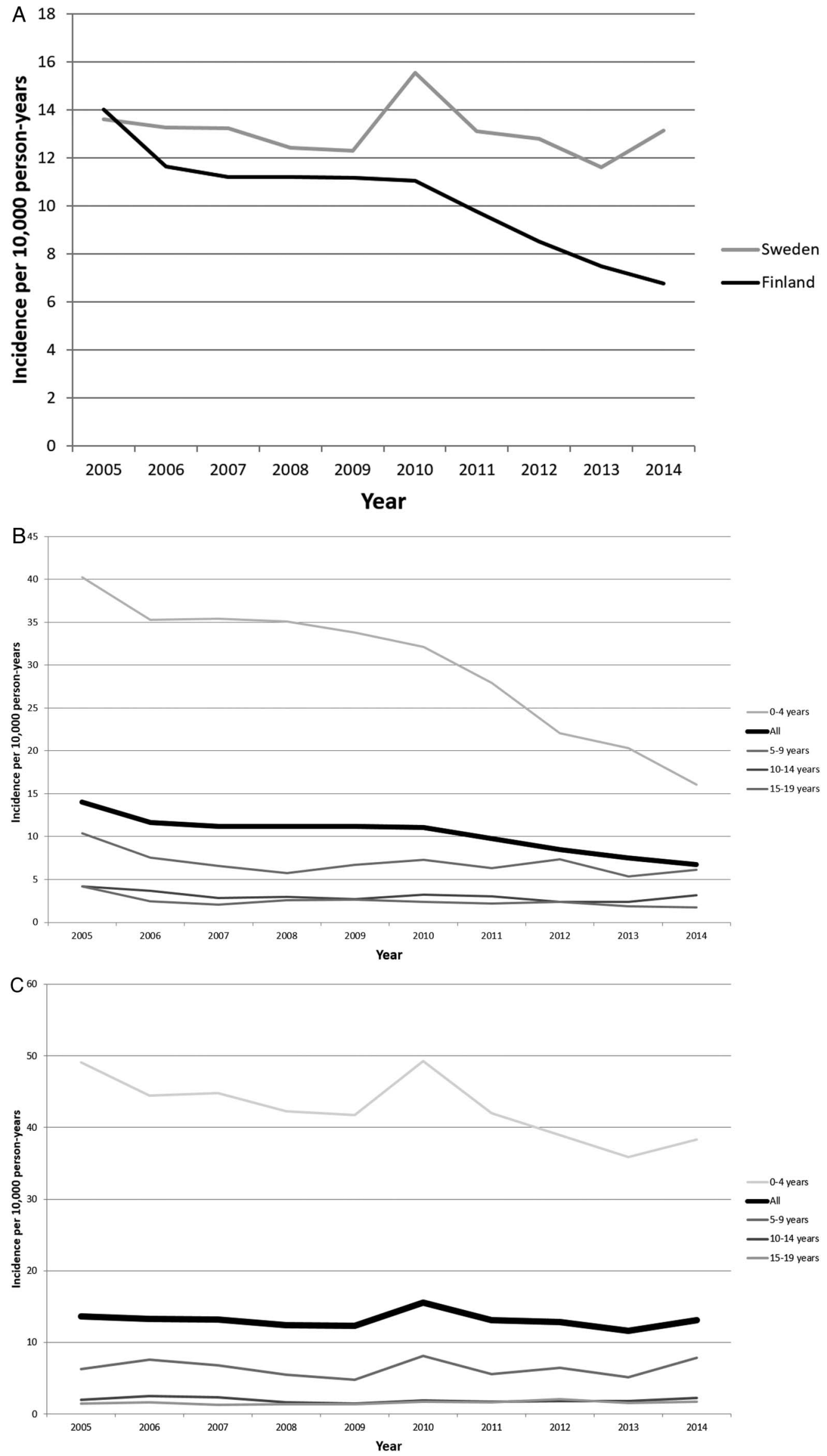

Figure 1 Incidence of hospitalisations for asthma per 10000 person-years among Finnish and Swedish children (aged 0-19 years) between 2005 and 2014. (A) Finland and Sweden. (B)Finland: age groups and total. (C) Sweden: age groups and total. 
Figure 2 Incidence of hospitalisations for small wheezers per 10000 person-years among Finnish and Swedish children (aged 0-4 years) between 2005 and 2014 (all=combined diagnosis $\mathrm{J} 21$ and J45-J46).

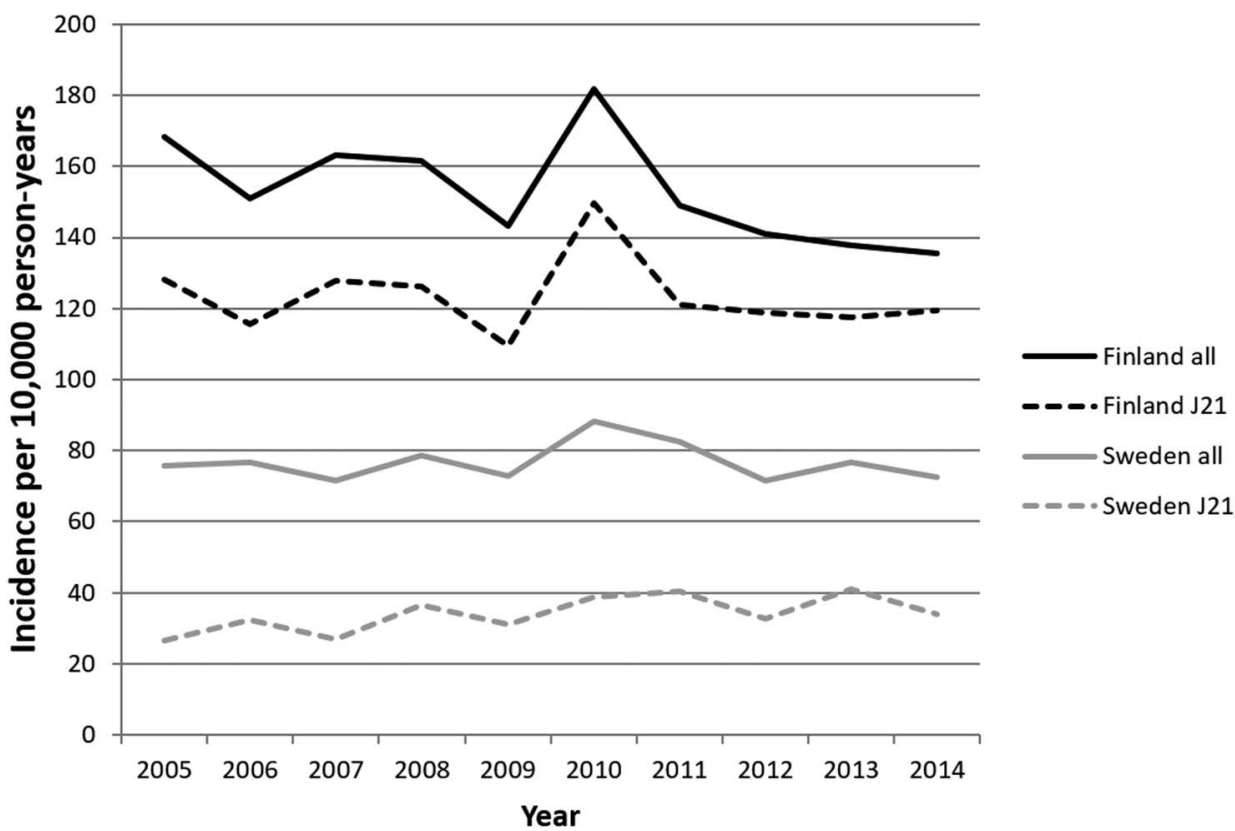

in both countries, the changes in paediatric asthma hospitalisation likely do not reflect changes in coding (eg, from asthma to wheezing). Notably, the incidence of hospital admissions due to wheezing is higher in Finland compared with Sweden. To our knowledge, there are no real differences in coding or diagnosing J21. We speculate that 'small wheezers' may be more commonly admitted to hospital in Finland, whereas more obstructive episodes and wheezing are treated in Swedish ED.

The change in hospitalisations noted in 2010 may be attributable to a high incidence of respiratory syncytial virus infections in both countries during that year. Also, the differences between countries may be due to differences between national guidelines. For example, the number of wheezing episodes required for an asthma diagnosis in young children differs between the countries. In Finland, Current Care Guidelines were updated first in 2010 and stricter guidelines were introduced in 2012. These changes may have affected the changes in hospitalisations due to asthma. ${ }^{11}$

In summary, asthma hospitalisations among Finnish and Swedish children aged 0-4 years are decreasing, especially in Finland. The reasons for the differences between countries are not completely clear, thus warranting future research on the subject.

\section{Juho E Kivistö, 1,2,3 Jennifer L P Protudjer, ${ }^{3,4}$ Jussi Karjalainen, ${ }^{1}$ Anna Bergström, ${ }^{3,4}$ Matti Korppi ${ }^{2}$}

${ }^{1}$ Allergy Centre, Tampere University Hospital, Tampere, Finland
${ }^{2}$ Tampere Center for Child Health Research, University of Tampere, Finland

${ }^{3}$ Institute of Environmental Medicine, Karolinska Institutet, Stockholm, Sweden

${ }^{4}$ Centre for Allergy Research, Karolinska Institutet, Stockholm, Sweden

Correspondence to Dr Juho Kivistö, Allergy Centre, Tampere University Hospital, PL 2000, Tampere 33521, Finland; juho.kivisto@uta.fi

Contributors JEK conceptualised and designed the study, designed the data collection instruments, carried out the analyses and drafted the initial manuscript, and approved the final manuscript as submitted. JLPP and $A B$ conceptualised the study, designed the data collection instruments, reviewed and revised the manuscript, and approved the final manuscript as submitted. JK conceptualised the study, reviewed and revised the manuscript, and approved the final manuscript as submitted. MK conceptualised and designed the study, reviewed and revised the manuscript, and approved the final manuscript as submitted.

Funding The project received funding from Tampere TB Foundation, Väinö and Laina Kivi Foundation, The Finnish Society of Allergology and Immunology, The Research Foundation of the Pulmonary Diseases and Paulo Foundation.

Competing interests None declared.

Provenance and peer review Not commissioned; externally peer reviewed.

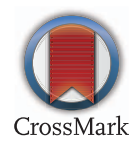

To cite Kivistö JE, Protudjer JLP, Karjalainen J, et al. Thorax 2018;73:185-187.

Received 16 November 2016

Revised 23 February 2017

Accepted 12 March 2017

Published Online First 3 April 2017

Thorax 2018;73:185-187.

doi:10.1136/thoraxjnl-2016-209739

\section{REFERENCES}

1 Worldwide variation in prevalence of symptoms of asthma, allergic rhinoconjunctivitis, and atopic eczema: ISAAC. The International Study of Asthma and Allergies in Childhood (ISAAC) Steering Committee. Lancet 1998;351:1225-32.

2 de Miguel-Diez J, Jimenez-Garcia R, Hernandez-Barrera $\mathrm{V}$, et al. National trends in hospital admissions for asthma exacerbations among pediatric and young adult population in Spain (2002-2010). Respir Med 2014;108:983-91.

3 Kivistö JE, Protudjer JL, Karjalainen J, et al. Hospitalisations due to allergic reactions in Finnish and Swedish children during 1999-2011. Allergy 2016;71:677-83.

4 Kocevar VS, Bisgaard H, Jonsson L, et al. Variations in pediatric asthma hospitalization rates and costs between and within Nordic countries. Chest 2004;125:1680-4.

5 Wettermark B, Hammar N, Fored CM, et al. The new Swedish Prescribed Drug Register--opportunities for pharmacoepidemiological research and experience from the first six months. Pharmacoepidemiol Drug Saf 2007;16:726-35

6 Aro S, Koskinen R, Keskimäki I.

Sairaalastapoistorekisterin diagnoosi-, toimenpide- ja tapaturmatietojen luotettavuus [Reliability of hospital discharge data concerning diagnosis, treatments and accidents]. Duodecim; laaketieteellinen aikakauskirja 1990;106:1443-50.

7 Hasegawa K, Tsugawa Y, Brown DF, et al. Childhood asthma hospitalizations in the United States, 2000-2009. J Pediatr 2013;163:1127-33 e3.

8 Wennergren G, Strannegard IL. Asthma hospitalizations continue to decrease in schoolchildren but hospitalization rates for wheezing illnesses remain high in young children. Acta Paediatrica 2002;91:1239-45.

9 Saynajakangas 0 , Valmari $\mathrm{P}$, Tuuponen $\mathrm{T}$, et al. Trends in hospitalization for childhood asthma in Finland in 1996-2004. Acta Paediatrica 2007;96:919-23.

10 Malmstrom K, Korhonen K, Kaila M, et al. Acute childhood asthma in Finland: a retrospective review of hospital admissions from 1976 to 1995. Pediatr Allergy Immunol 2000;11:236-40.

11 Haahtela T, Lehtimaki L, Ahonen E, et al. [Update on current care guidelines: asthma]. Duodecim 2013;129:994-5. 